\title{
Factores asociados
}

\section{a la utilización de servicios de atención ambulatoria en México: un análisis de los proveedores}

\author{
Factors Associated with the Use \\ of Ambulatory Care in Mexico:
} An Analysis of Health Service Providers

\section{Fatores associados à utilização de serviços de atenção ambulatória no México: uma análise dos fornecedores}

Hugo Nathanael Lara Figueroa, $\mathrm{PhD}^{1 *}$

Edith Miriam García Salazar, PhD²

Recibido: . $^{\circ}$ de abril de 2019 - Aceptado: 23 de julio de 2020

Doi: https://doi.org/10.12804/revistas.urosario.edu.co/revsalud/a.9790

Para citar este artículo: Lara Figueroa HN, García Salazar EM. Factores associados a la utilización de servicios de atención ambulatoria en México: un análisis de los provedores. Rev Cienc Salud. 2020;18(3):1-17. https://doi.org/10.12804/revistas.urosario.edu.co/ revsalud/a.9790

\section{Resumen}

Introducción: el estudio de la salud como un bien económico señala que la demanda de servicios para la salud deriva de la necesidad percibida de una persona que solicita atención por motivos de prevención o curación. Para que esta se convierta en utilización deben existir, al mismo tiempo, prestadores que los proporcionen. Analizar las características de los distintos proveedores resulta útil para conocer cómo influyen en la demanda, así como en la manera en que puede mejorarse la atención. El objetivo es identificar las características de la oferta de servicios para la salud que inciden en la utilización de atención ambulatoria de México. Materiales y métodos: se realizó un estudio transversal con información de la Encuesta Nacional de Salud y Nutrición (Ensanut) de 2018 y se elaboró un modelo de regresión logístico condicional que incluyó las características de los proveedores. Se simularon escenarios para mostrar cambios en la probabilidad de la utilización diferenciada ante cambios en las variables de mayor impacto en el modelo. Resultados: las variables incorporadas en el modelo muestran una incidencia en la elección de proveedores; el precio del servicio presentó un impacto menor a los hallazgos de otros estudios, lo que sugiere que la atención ambulatoria pública se asemeja al comportamiento de un bien

1 Tecnológico de Estudios Superiores de Ecatepec (México).

Autor de correspondencia: hugolara@tese.edu.mx orciD: https://orcid.org/0000-0003-2832-8422

2 El Colegio del Estado de Hidalgo (México). orciD: https://orcid.org/0000-0003-1141-0147 
inferior. Conclusión: las características de los proveedores tienen impactos similares al de algunas variables sociodemográficas y económicas documentadas en diversos estudios.

Palabras clave: atención ambulatoria; utilización; demanda; servicios de salud.

\section{Abstract}

Introduction: The study of health as an economic good indicates that the demand for health services is derived from the perceived need of a person seeking care for prevention or cure. To this end, providers of these services are required simultaneously. Analysis of the characteristics of different health service providers is useful to understand how they influence demand as well as how care can be improved. The objective of this study was to identify the characteristics of health service providers that affect the use of ambulatory care in Mexico. Materials and methods: A cross-sectional study was conducted using information from the 2018 National Health and Nutrition Survey (Ensanut), and a conditional logistic regression model was prepared, which included the characteristics of the providers. Scenarios were simulated to show changes in the probability of differentiated use, given that there were changes in the variables with the greatest impact on the model. Results: The variables incorporated in the model demonstrated an incidence in the choice of providers. Moreover, the price of the service had less impact compared with the findings of other studies, suggesting that public outpatient care resembles the characteristics of an inferior good. Conclusion: The characteristics of health service providers have impacts similar to those of some sociodemographic and economic variables documented in various studies.

Keywords: ambulatory care; utilization; demand; health services.

\section{Resumo}

Introdução: o estudo da saúde como um bem econômico assinala que a demanda de serviços para a saúde deriva da necessidade percebida de uma pessoa que solicita atenção por motivos de prevenção ou cura. Para que esta se torne em utilização devem existir, ao mesmo tempo, prestadores que os proporcionem. Analisar as características dos distintos fornecedores resulta útil para conhecer como estes influem na demanda, assim como na maneira em que pode melhorar-se a atenção. $O$ objetivo é identificar as características da oferta de serviços para a saúde que incidem na utilização de atenção ambulatória do México. Materiais e métodos: se realizou um estudo transversal com informação do Inquérito Nacional de Saúde e Nutrição (Ensanut) de 2018 e se elaborou um modelo de regressão logístico condicional incluindo as características dos fornecedores; se simularam cenários para mostrar mudanças na probabilidade da utilização diferenciada, ante mudanças nas variáveis de maior impacto no modelo. Resultados: as variáveis incorporadas no modelo mostram uma incidência na eleição de fornecedores; o preço do serviço apresentou um impacto menor aos resultados de outros estudos, o que sugere que a atenção ambulatória pública se assemelha ao comportamento de um bem inferior. Conclusão: as características dos fornecedores têm impactos similares ao de algumas variáveis sociodemográficas e econômicas documentadas em diversos estudos.

Palavras-chave: atenção ambulatória; utilização; demanda; serviços de saúde.

\section{Introducción}

$\mathbf{E}_{\text {servicios suministrados por instituciones de seguridad social — dirigidos a aquella pobla- }}^{\text {n México existe una oferta de servicios para la salud que se clasifica en tres tipos: } 1 \text { los }}$ ción asalariada afiliada a instituciones como: el Instituto Mexicano del Seguro Social (IMss), 
el Instituto de Seguridad y Servicios Sociales de los Trabajadores del Estado (ISSSTE), servicios de salud otorgados para los trabajadores de Petróleos Mexicanos y la Secretaría de Marina-; 2) los servicios para la salud de carácter público — que atienden a la población abierta (sin acceso a la seguridad social) mediante el Sistema de Protección Social en Salud a través del Seguro Popular en Salud (ahora Instituto Nacional para el Bienestar), y 3) el sector privado, en el que cualquier persona puede atenderse mediante pagos directos o por la contratación de un seguro contra gastos médicos $(1,2)$.

Si bien, y a pesar de que la gente puede recibir de forma gratuita atención médica de carácter pública o que las personas pueden asistir a los servicios a los cuales se encuentran afiliados, durante los últimos años se ha observado un aumento en la utilización de servicios privados en México, lo cual ha llevado a que muchos de los hogares sufraguen erogaciones por encima de su capacidad de pago (3). Ello provoca gastos catastróficos y empobrecedores que ponen en riesgo su calidad de vida y bienestar ante la necesidad de recurrir a fuentes de financiamiento alternativas, como venta de activos, endeudamiento o sacrificio de otro tipo de bienes de consumo e inversión (alimentación, educación, entre otros) (3-10).

Desde esta perspectiva, la economía de la salud ha sido un referente teórico y empírico para explicar por qué se producen cambios en la utilización de servicios de atención médica. El empleo de modelos de comportamiento desarrollados a partir de estimaciones econométricas ha permitido identificar cuáles aspectos inciden para que una persona, ante un evento de enfermedad (curación), o con la intención de mantener su estado de salud (prevención), decida atenderse (o no) y en qué lugar hacerlo (11-20).

En el caso mexicano, los estudios se han abordado principalmente desde el enfoque de la demanda, apoyándose en los supuestos de racionalidad económica, la utilidad individual y las preferencias de estos en la elección de los servicios disponibles (5-8,10,20-27). Este enfoque se deriva de la teoría económica tradicional del consumidor, en la cual el individuo es el principal agente determinador de la demanda de servicios para la salud, pero con restricciones originadas de las características de los proveedores que los suministran. El poder explicativo de este enfoque ha resultado vital cuando se trata de conocer el impacto provocado por los aspectos geográficos, económicos y culturales en la utilización de tales servicios. Al respecto, podemos mencionar que mediante este tipo de estudios se conoce, por ejemplo, que las personas de un hogar, región específica y con ciertos ingresos estarían más interesadas en utilizar servicios a los cuales están afiliados, o bien, acudir a aquellos donde los costos de traslado son menores, por mencionar algunos.

Si bien las contribuciones de estos estudios han resultado importantes para identificar por qué se dan cambios en la demanda, su aporte es limitado cuando la utilización es diferenciada en individuos o familias que cuentan con perfiles sociodemográficos o económicos similares, con lo cual se puede asumir que las preferencias de estos se encuentran determinadas 
también por las características de los servicios a los cuales acuden cuando requieren algún tipo de atención.

Debido a lo anterior se han desarrollado estudios que emplean modelizaciones en las que se incluyen características de las alternativas disponibles. Entre ellos destacan los modelos logístico condicional, los anidados o los modelos de efectos mixtos $(11-14,17,18)$.

Con el empleo de este tipo de estimaciones se identifican aquellos factores que los consumidores (utilizadores) consideran cuando eligen una opción entre más de dos alternativas. Se trata de generalizaciones de los modelos de regresión logit y probit con la diferencia de que mientras los segundos suponen independencia entre las distintas alternativas posibles, es decir, que las probabilidades de las funciones de utilidad condicional no están correlacionadas a través de las alternativas - las opciones A y B son bienes normales (ninguna se considera un bien sustituto) y el individuo puede elegir cualquier alternativa de manera independiente-; los primeros identifican los factores que determinan la elección de un individuo entre las opciones que se ofrecen, suponiendo que estas son dependientes entre sí -la opción A (bien sustituto) puede ser elegida debido a que el usuario cuenta con restricciones para elegir la opción B-. La evidencia empírica obtenida de este tipo de modelizaciones ha contribuido a identificar que, además de las variables sociodemográficas y económicas (severidad de la enfermedad, edad, sexo, estado civil, la actitud individual del usuario, ingreso-gasto) de los individuos, las características de los proveedores incidirían en la elasticidad del precio de la demanda (cambios en la utilización de un tipo de proveedor cuando cambia su precio), pero también, en la elección de algún servicio en función a sus atributos $(12,14,17,18,28)$.

Desde esta perspectiva, el objetivo del presente estudio consiste en identificar las características de la oferta de servicios para la salud que inciden en la utilización de atención ambulatoria en el caso mexicano.

\section{Materiales y métodos}

ara conocer los factores asociados a los cambios en el uso de servicios de atención ambu-
latoria, se empleó un modelo logístico condicional mediante una modelización con variables discretas. Estos modelos constituyen un instrumento estadístico cuando se cuenta con una variable dependiente cualitativa con dos (binomial) o más valores posibles (multinomial) no jerarquizados (nominal) y, al menos, una variable independiente que puede ser cuantitativa (covariable) o categórica (nominal u ordinal).

Es útil cuando se quiere conocer la probabilidad de que ocurra o no algún evento de interés a partir de una estimación que identifica, por ejemplo, la incidencia (magnitud mediante coeficientes) que una variable predictora (independiente/explicativa) tiene en la 
elección de los distintos proveedores de servicios para la salud. Con ello se sabe qué y cuáles variables (características de los servicios) aumentan o disminuyen la probabilidad de que una persona decida acudir a algún tipo de proveedor de servicios para la salud.

En el modelo desarrollado en este estudio, la utilidad que un individuo $n$ recibe de la elección de acudir a alguna institución de salud disponible $i$, dado un conjunto alternativas $i=1, \ldots I$, se representa mediante una función indirecta de la utilidad lineal con la siguiente forma:

$$
U_{n i}=\beta^{\prime} X_{n i}+\varepsilon_{n i}
$$

Donde:

$\beta^{\prime} X_{n i}$ es la parte no estocástica de la utilidad indirecta obtenida cuando el individuo utiliza la alternativa $i$.

$X_{n i}$ son los atributos observados que caracterizan las alternativas disponibles (disponibilidad de material clínico, medicamentos, por mencionar algunas) para el utilizador.

$\beta^{\prime}$ es el vector de coeficientes (magnitud/incidencia) estimados para cada uno de los atributos observados de $X_{n i}$.

$\varepsilon_{n i}$ es el término de error que representa la variación en las preferencias de los distintos utilizadores (variable o atributos no incorporados al modelo).

Si se asume que un individuo siempre elegirá la alternativa en la cual maximiza su utilidad (proveedor de su preferencia), la probabilidad $\pi_{n i}$ de la elección de la alternativa $i$ será mayor que las del resto de las alternativas, misma que al simplificarse queda expresada como:

$$
\pi_{n i}=\frac{e^{\beta^{\prime} X_{n i}}}{\sum_{j=1}^{I} \beta^{\prime} X_{n j}}
$$

Finalmente, al transformar los coeficientes en probabilidades, se identifican aquellas características de los proveedores capaces de influir en la elección de las opciones disponibles; pero también, en los atributos que, para el caso de los servicios que suministran las instituciones de salud públicas y de seguridad social, pueden mejorarse, lo que reduce, como contraparte, la probabilidad de incurrir en un gasto catastrófico y empobrecedor ante la utilización de servicios privados.

La variable dependiente se modeló de manera discreta (categórica) con tres valores posibles: utilizar servicios de salud públicos, de seguridad social o privados. La categoría de referencia (servicios privados) incluyó a individuos que utilizaron servicios para la salud en instituciones privadas (farmacias similares y hospitales de primer nivel: Hospital Ángeles, Médica Sur, entre otros); para los servicios de seguridad social se consideraron a todas aquellas personas que acudieron a servicios como el Instituto Mexicano del Seguro Social, el Instituto de Seguridad y Servicios Sociales de los Trabajadores del Estado, servicios 
de salud otorgados para los trabajadores de Petróleos Mexicanos y la Secretaría de Marina. Finalmente, en los servicios de salud públicos se incluyó a quienes se atendieron en instituciones de la Secretaría de Salud: Imss Oportunidades, Seguro Popular y el Sistema Nacional para el Desarrollo Integral de la Familia.

Respecto con las variables explicativas, se seleccionaron aquellas preguntas que caracterizan a los proveedores de servicios para la salud y que forman parte de las secciones III-VII del cuestionario de utilizadores de la Encuesta Nacional de Salud y Nutrición (Ensanut) 2018 (29). Adicionalmente, se incluyó la variable afiliación, relacionada con las características del utilizador. Respecto a la estructura de las variables explicativas, se describen en la tabla 1.

Tabla 1. Variables explicativas

\begin{tabular}{|c|c|}
\hline Variable & Codificación \\
\hline Afiliación & $\begin{array}{l}\text { Variable categórica que toma } 7 \text { valores: } 0 \text { si el hogar no se encuentra afiliado a ningún ser- } \\
\text { vicio para la salud, } 1 \text { si está adscrito a alguna institución de seguridad social, } 2 \text { si cuenta } \\
\text { con aseguramiento público, } 3 \text { cuando pertenece a algún sistema privado, } 4 \text { cuando el uti- } \\
\text { lizador cuenta con cobertura pública y de seguridad social, } 5 \text { cuando el utilizador cuenta } \\
\text { con una doble cobertura de servicios de seguridad social, } 6 \text { cuando la doble cobertura se } \\
\text { da a través de servicios de seguridad social y públicos y } 7 \text { cuando se encuentra afiliado a } \\
\text { servicios privados y públicos. }\end{array}$ \\
\hline $\begin{array}{l}\text { Precio de la } \\
\text { consulta }\end{array}$ & $\begin{array}{l}\text { Variable continua que indica el desembolso sufragado por quien solicitó servicios de aten- } \\
\text { ción médica ambulatoria. }\end{array}$ \\
\hline $\begin{array}{l}\text { Disponibilidad } \\
\text { de medicamentos }\end{array}$ & $\begin{array}{l}\text { Variable categórica con } 4 \text { valores: } 0 \text { cuando el utilizador no requirió medicamentos, } \\
1 \text { cuando el utilizador requirió medicamentos y los obtuvo en el mismo lugar de aten- } \\
\text { ción, } 2 \text { cuando los obtuvo en otro lugar de la atención, pero en la misma institución } \\
\text { y } 3 \text { cuando los obtuvo en otro lugar. }\end{array}$ \\
\hline $\begin{array}{l}\text { Disponibilidad de } \\
\text { material clínico }\end{array}$ & $\begin{array}{l}\text { Variable categórica con } 4 \text { valores: } 0 \text { cuando el utilizador no requirió exámenes de labo- } \\
\text { ratorio, } 1 \text { cuando sí los requirió y los obtuvo en el mismo lugar de atención, } 2 \text { cuando } \\
\text { se realizó exámenes médicos en otro lugar de la atención pero en la misma institución } \\
\text { y } 3 \text { cuando se los realizó en otro lugar. }\end{array}$ \\
\hline $\begin{array}{l}\text { Atención } \\
\text { mediante cita }\end{array}$ & $\begin{array}{l}\text { Variable categórica con dos valores: } 0 \text { cuando el utilizador no recibió atención mediante } \\
\text { cita programada y } 1 \text { cuando lo hizo mediante cita. }\end{array}$ \\
\hline $\begin{array}{l}\text { Elección del lugar } \\
\text { de atención }\end{array}$ & $\begin{array}{l}\text { Variable categórica con dos valores posibles: } 0 \text { cuando no eligió el lugar de atención } \\
\text { y } 1 \text { cuando sílo hizo. }\end{array}$ \\
\hline $\begin{array}{l}\text { Diagnóstico sobre } \\
\text { la enfermedad al } \\
\text { paciente }\end{array}$ & $\begin{array}{l}\text { Variable categórica con dos valores: } 0 \text { cuando el médico no informó al usuario sobre la } \\
\text { enfermedad del paciente y } 1 \text { cuando sí lo hizo. }\end{array}$ \\
\hline
\end{tabular}

Para estimar la demanda por servicios médicos curativos se utilizó información correspondiente a la Ensanut 2018 (29). La encuesta es de cobertura nacional y cuenta con información de 50000 viviendas. A efectos de la estimación fue necesario restringir la muestra, a modo de incluir solamente a individuos que tuvieron necesidad de atención ambulatoria curativa. También se excluyeron casos con información incompleta. Con dichas acotaciones se analizaron, de un universo total de 8757 cuestionarios de utilizadores, 7958 casos que, con base en los factores de expansión, representan 7306793 individuos, que presentaron un 
evento de enfermedad y que acudieron a alguna de las instituciones de salud distribuidas en toda la república mexicana.

Respecto al tratamiento de la información, se hicieron pruebas de ajuste en aquellas variables que estudios previos han encontrado con comportamientos no lineales y se optó por mantener la variable costo de la atención (precio de la consulta) como continua, debido a que con ello hubo un mejor ajuste. En el caso de las variables categóricas, se mantuvieron los valores originales, por lo que fue posible obtener coeficientes individuales para cada tramo de dichas variables. Finalmente, los coeficientes fueron transformados en cambios en las probabilidades estimadas a partir de impactos marginales ante cambios en las categorías.

\section{Resultados}

Como se observa en la tabla 2, los resultados muestran valores estadísticamente significativos y con coeficientes de diferente magnitud. Las variables afiliación, disponibilidad de material clínico, diagnóstico de la enfermedad, disponibilidad de medicamentos y atención mediante cita cuentan con los valores más altos.

Así, mientras los resultados corroboran los hallazgos, en estudios previos para el caso de las variables precio de la atención, afiliación y disponibilidad de medicamentos (5-10,2325,27,30-42), la explicación de la enfermedad (diagnóstico), atención mediante cita (37) y disponibilidad de material clínico también son factores que influyen en la utilización diferenciada. Un caso interesante se observó en la variable precio, que presentó un valor considerado por debajo de lo esperado respecto con otros estudios. Esta situación bien puede ser atribuida a que, en el caso de servicios ambulatorios públicos, el precio por la atención tiene un comportamiento similar al de un bien inferior, donde al aumentar el precio (o los costos indirectos asociados a su utilización), disminuye la cantidad demandada, pero en menor medida al de otras variables (afiliación, atención mediante cita, por mencionar algunas). 
Tabla 2. Probabilidad de utilizar servicios de atención ambulatoria por tipo de institución (servicios privados como referencia)

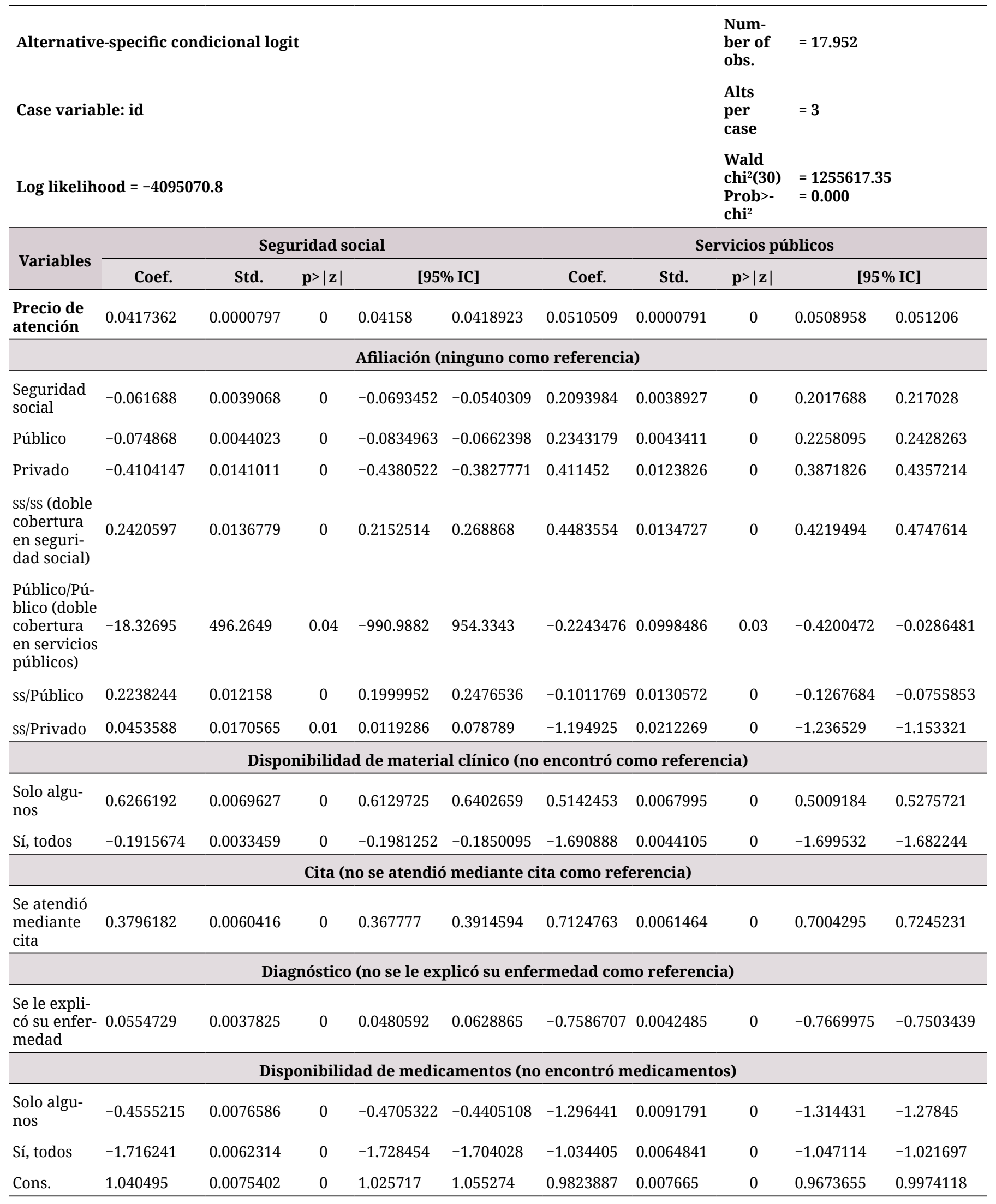


A fin de facilitar la interpretación de los resultados, se transformaron en probabilidades aquellos atributos que registraron los valores más altos. Para ello, se estimaron impactos marginales cambiando los valores de las categorías relacionadas con: disponibilidad de medicamentos y material de laboratorio, afiliación, consulta programada y diagnóstico de la enfermedad, manteniendo fijos los valores promedio en el resto de variables incorporadas al modelo general.

La figura 1 muestra el efecto que produce la disponibilidad de medicamentos y material clínico en las instituciones de salud. Como se observa, los servicios de seguridad social presentan nulas probabilidades de ser utilizados cuando no se cuenta con medicamentos; mientras que en los servicios privados existe una alta probabilidad de utilización para las tres categorías. Por su parte, las probabilidades de acudir a servicios públicos incrementan cuando existen algunos o todos los medicamentos. Los resultados obtenidos sugieren que la disponibilidad de estos es un elemento importante en servicios de carácter público y social; en los servicios privados, aunque se observan diferenciales importantes, las probabilidades de uso siempre son las más altas en comparación al resto de los proveedores. Un caso similar ocurre con la disponibilidad de material clínico, en que las probabilidades de utilización más altas se dan en los servicios privados; en los servicios de seguridad social, es prácticamente nula en las tres categorías, mientras que en los servicios públicos las probabilidades pueden considerarse altas cuando la atención no requiere exámenes de laboratorio, o bien cuando existe todo el material clínico.

Disponibilidad de medicamentos

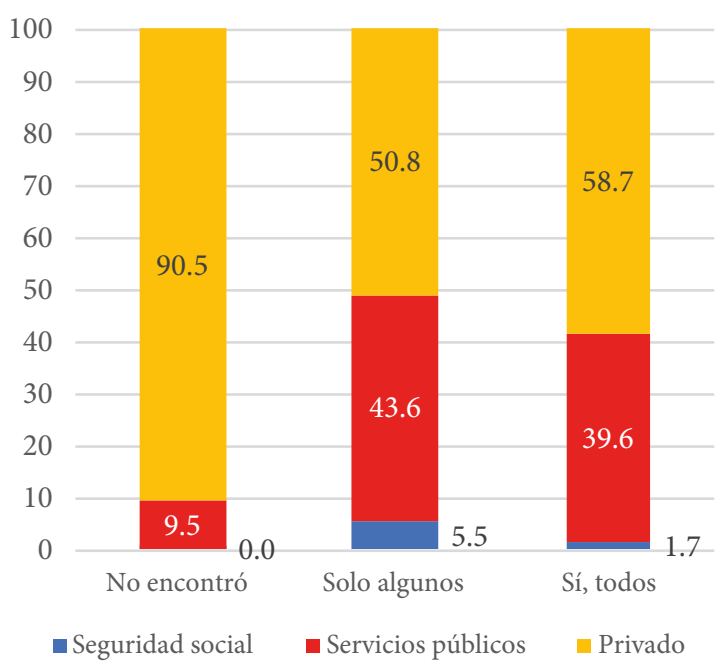

Disponibilidad de material clínico

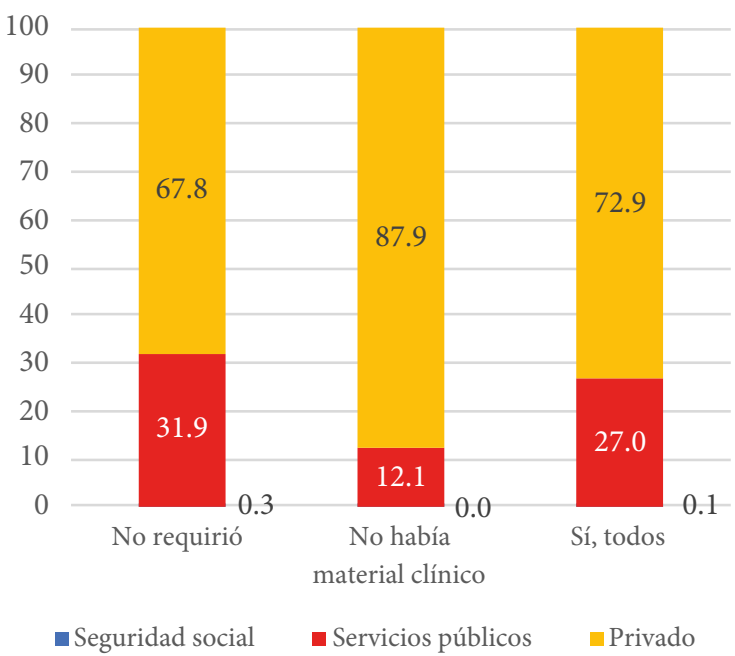

Figura 1. Probabilidad de utilizar servicios de seguridad social, públicos y privados ante cambios en la disponibilidad de medicamentos y material clínico

Fuente: elaboración propia. 
La figura 1 muestra que el no encontrar medicamentos en las instituciones de atención tiene un efecto importante en el tipo de servicios de salud que los usuarios utilizan; la prevalencia probabilística en servicios privados se mantiene como la más alta de todas las instituciones, lo cual sugiere que una parte importante de quienes presentan eventos de enfermedad de primer nivel tiende a acudir a servicios de seguridad social y, en menor medida, a servicios públicos, cuando resulta posible encontrar, al menos, algunos medicamentos.

Los cambios en las probabilidades por tipo de afiliación arrojan resultados interesantes. La figura 2 muestra que los servicios públicos no han tenido el impacto esperado en términos de utilización, ya que los emplean menos de una tercera parte del total de usuarios que no cuentan con cobertura alguna en salud; mientras que la atención de estos se concentra principalmente en instituciones privadas, cifra muy similar para el caso de quienes cuentan con una sola cobertura pública. También se observa que este tipo de servicios solamente es usado cuando se cuenta con una doble cobertura en servicios públicos. No obstante, y salvo para esta categoría, la utilización de servicios privados permanece como la más preferida cuando se trata de atención ambulatoria. En este sentido, si bien la cobertura universal puede tener un efecto financiero protector, es menor al esperado.

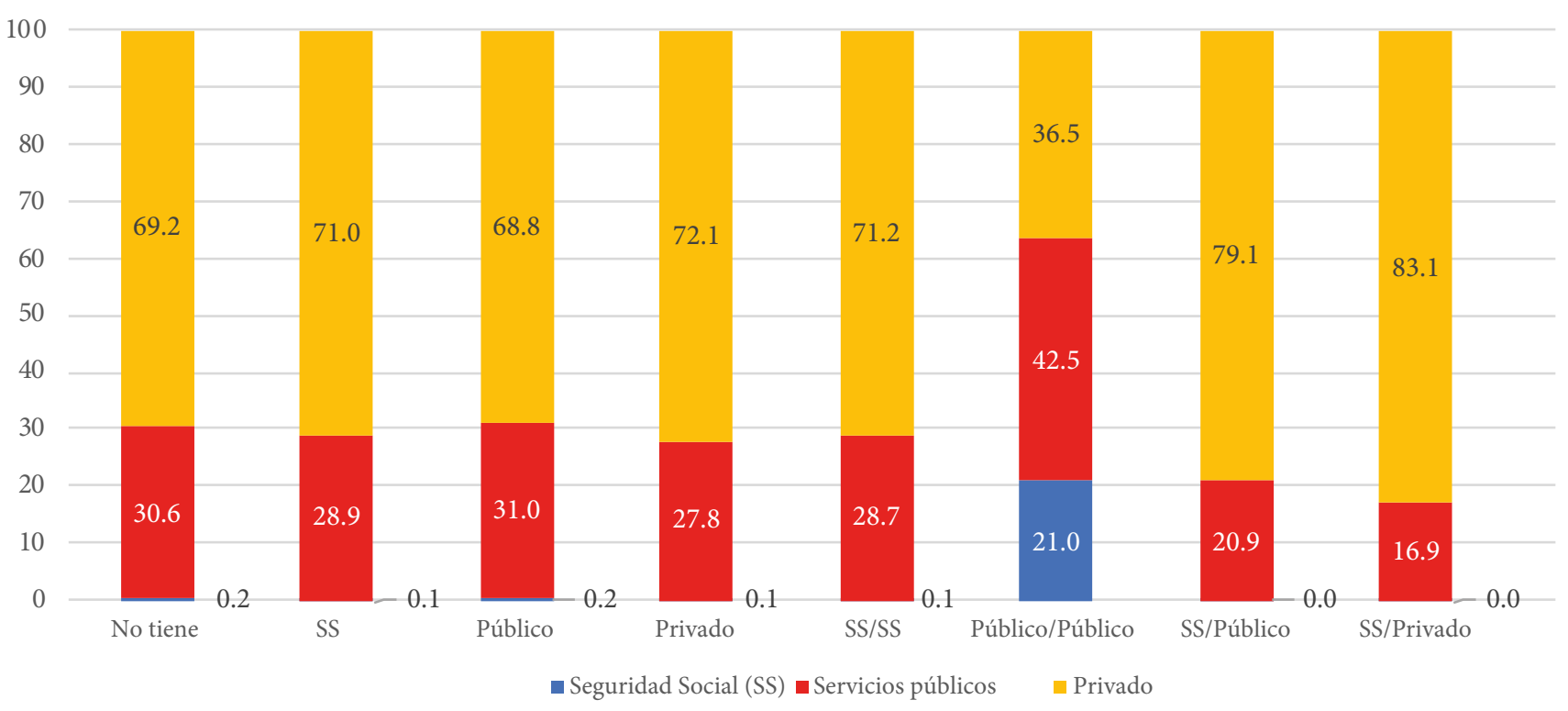

Figura 2. Probabilidad de utilizar servicios de seguridad social, públicos y privados por tipo de aseguramiento

Finalmente, y respecto al tipo de consulta y explicación de la enfermedad, los resultados muestran que acudir a recibir atención médica con cita es un elemento importante, salvo en los servicios privados, para quienes demandan servicios con cualquier tipo de proveedor, ya que las probabilidades son mayores que cuando acuden sin atención programada (figura 3). Lo anterior bien puede deberse al hecho de que en México es muy habitual encontrar largos tiempos de espera para concertar una cita, o bien para ser atendidos cuando la atención no ha 
sido programada, particularmente cuando es en instituciones públicas o de seguridad social (43). Respecto a la variable diagnóstico, los resultados indican que cuando los médicos explican el estado de salud a sus pacientes, las probabilidades de acudir a cualquier tipo de servicio son mayores que cuando no lo hacen. Este hecho resalta la importancia que los usuarios le dan al conocimiento sobre su padecimiento, al punto de que esto puede garantizar que los enfermos utilicen cualquier proveedor o regresar en caso de que sea necesario.
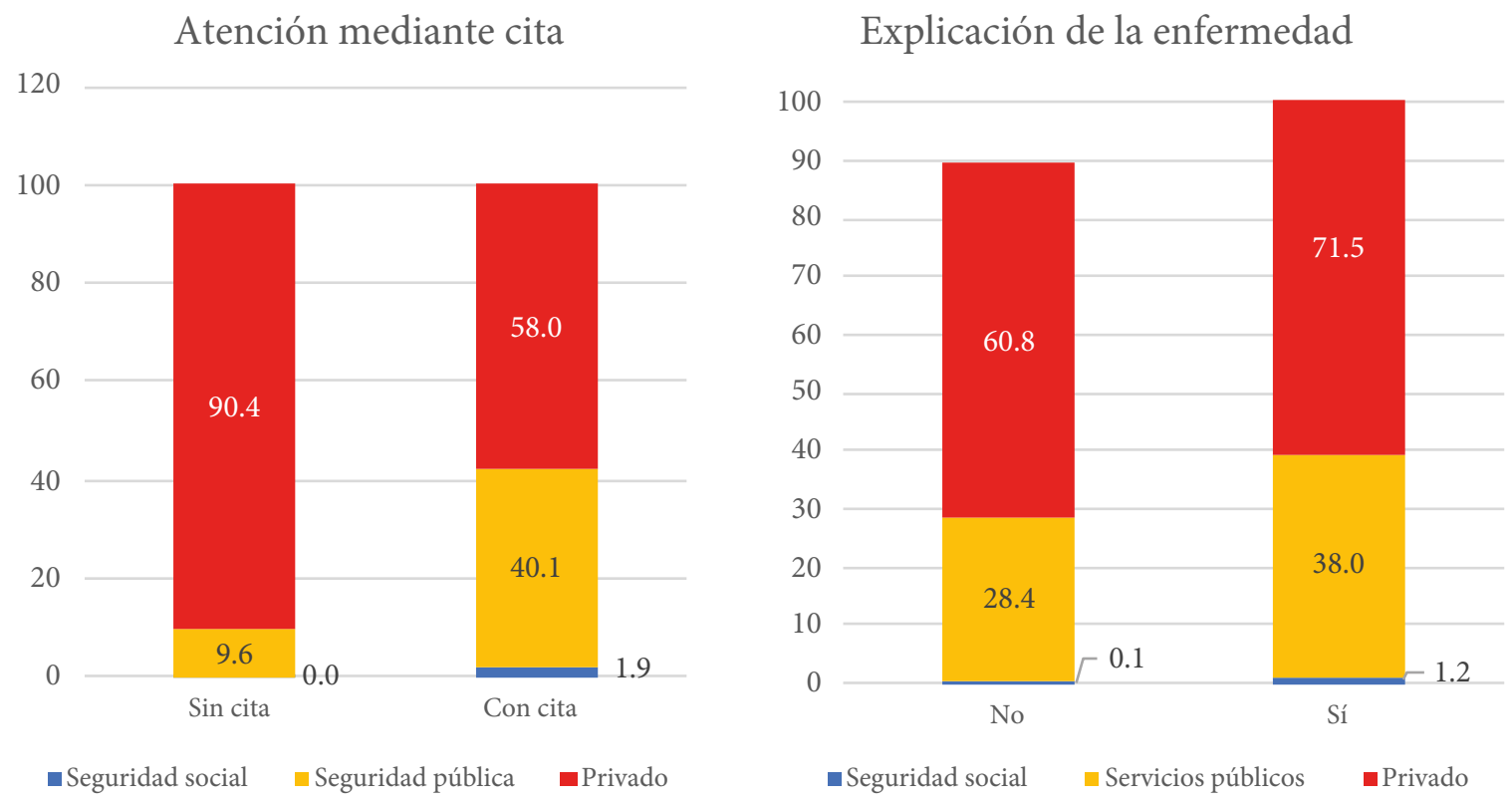

Figura 3. Probabilidad de utilización de los servicios de salud por tipo de consulta y explicación de la enfermedad

\section{Discusión}

\section{L}

os resultados obtenidos en el modelo logístico condicional muestran el grado y el tipo probabilidad de utilizar algún tipo de servicios para la salud de México. El hecho de que los coeficientes obtenidos en cada categoría muestren signos positivos y negativos indica un impacto diferenciado de sus atributos en la categoría de referencia (servicios privados) de la variable dependiente.

En el caso del precio de la consulta, los valores presentaron signos positivos para los casos de seguridad social y pública, con lo cual se corroboran los hallazgos en estudios similares, en los cuales se muestra el peso de este $(23-25,28)$. No obstante, también se pudo documentar que el impacto es menor al de otras variables incluidas.

Por su parte, la variable afiliación muestra que la probabilidad de acudir a servicios privados aumenta cuando: la persona o el hogar se encuentra afiliado a servicios de seguridad social, público, o con una cobertura doble en servicios público-público, esto para el caso de los 
utilizadores de servicios de seguridad social. Para quienes se atendieron en servicios públicos, las probabilidades de utilizar servicios privados aumentan en los casos de doble cobertura en servicios público-público, seguridad social-público y seguridad social-privado. Al igual que en el caso anterior, los resultados son consistentes con los estudios que documentan cómo la afiliación favorece de manera general la utilización de servicios para la salud y como mecanismo de protección financiera al evitar gasto de bolsillo en el que los utilizadores podrían incurrir cuando acuden a servicios privados (1,7,10,38,44-47). Sin embargo, resalta el hecho de que dicho impacto es diferenciado, por lo que la extensión de la cobertura universal en salud implementado en nuestro país no garantiza un efecto protector en todos los casos, ya que las personas sin aseguramiento y los que cuentan con una cobertura simple en servicios públicos tienen mayores probabilidades de atenderse en instituciones privadas.

Respecto a las variables diagnóstico de la enfermedad y atención programada, se encontró que las probabilidades son mayores indistintamente del proveedor cuando se explica la enfermedad y cuando la atención fue programada. A diferencia de las variables anteriores, los resultados no se pueden comparar con otros estudios, por lo que estos pueden considerarse un hallazgo que contribuye al diseño de estrategias y líneas de acción en materia de política pública para mejorar la atención en instituciones de salud de carácter público y de seguridad social.

En cuanto a la disponibilidad de medicamentos, se logró documentar que tanto para los servicios de seguridad social como para los públicos, las probabilidades de usarlos son mayores cuando se encuentran algunos o todos los medicamentos; mientras que para el caso de los servicios privados la disponibilidad no es tan importante. Los resultados obtenidos son similares a los realizados en otros estudios, donde se muestra la importancia que tiene la disponibilidad de medicamentes en la utilización y como mecanismo para prevenir gastos de bolsillo $(23,24,48,49)$.

Por último, la variable disponibilidad de material para exámenes de laboratorio muestra en todas las categorías que las probabilidades de acudir a servicios privados son siempre las más altas; mientras que en el caso de los servicios públicos esto solamente ocurre cuando los utilizadores no requieren material clínico para la toma de exámenes o cuando se requiere y está disponible en la unidad de atención. Un caso interesante se observa en los casos de servicios de seguridad social, donde la utilización prácticamente es nula para todas las categorías. Si bien no existen otros estudios relacionados de manera directa con la disponibilidad de este tipo de equipamiento, algunas investigaciones han podido documentar cómo la falta de material disponible puede provocar costos indirectos (casi un $20 \%$ del total de gastos sufragados por motivos de atención curativa) asociados a gasto en medicamentos sin receta, material para curación en servicios de medicina alternativa, aparatos ortopédicos y terapéuticos, así como cambios en la demanda de servicios para la salud a los cuales los utilizadores normalmente acuden (48).

Los hallazgos obtenidos muestran que las características de los proveedores impactan de forma similar en los aspectos sociodemográficos, económicos, epidemiológicos y culturales 
de los individuos, al punto de que estos pueden limitar el acceso y utilización de servicios ambulatorios de carácter público y social. En este sentido, la política para la salud en nuestro país deberá emplear mecanismos que garanticen no solamente la afiliación a un servicio de salud (público o de seguridad social), sino también que este suministre información relevante a sus pacientes sobre su enfermedad, citas oportunas y programadas, una mayor disponibilidad de medicamentos esenciales, material clínico necesario, entre otras. Así, la escasa o nula disponibilidad de estos atributos en los proveedores de salud deben ser considerados barreras importantes en el acceso que minimizan el efecto protector que tiene la adscripción a este tipo de servicios en términos económicos, lo cual puede ampliar las brechas financieras de los hogares en cuanto a su capacidad de pago, haciendo inequitativo el sistema de salud mexicano.

No obstante lo anterior, las estrategias preventivas serán siempre más efectivas, si lo que se quiere no es solo prevenir el gasto catastrófico o empobrecedor, sino garantizar el derecho a la salud y mejorar la calidad de vida y el bienestar de los mexicanos.

En suma, se requieren esfuerzos adicionales. El desafío pendiente más importante para México; ya no es solamente la extensión y afiliación a servicios de salud públicos y de seguridad social, sino también el rediseño de la política pública, centrada en mejorar los indicadores de estructura (disponibilidad de material clínico y de medicamentos) y proceso (explicación de la enfermedad y atención sin cita previa), a fin de que los grupos más vulnerables no tengan que acudir a servicios privados, evitando con ello sufragar erogaciones por encima de su poder adquisitivo.

\section{Contribución de los autores}

\footnotetext{
mbos autores contribuyeron en los apartados teórico, metodológico y en la redacción del Lartículo.
}

Edith Miriam García Salazar: antecedentes, análisis, procesamiento y estimación del modelo realizado, revisión biliográfica y conclusiones del estudio.

Hugo Nathanael Lara Figueroa: elección del tema, antecedentes, marco teórico, planteamiento de objetivo, selección y estimación del modelo econométrico, depuración de base de datos, estimación de probabilidades y discusión y conclusiones del estudio.

\section{Conflicto de intereses}

Ninguno declarado. 


\section{Referencias}

1. Gómez-Dantés O, Sesma S, Becerril VM, Knaul FM, Arreola H, Frenk J. Sistema de salud de México. Salud Publica Mex. 2011 Mar 7;53(Supl 2):s-220-32.

2. Zúñiga M, Carrillo G, Fos P, Gandek B, Medina M. Evaluación del estado de salud con la Encuesta SF-36: resultados preliminares en México. Salud Publica Mex. 1999;41(2):110-8.

3. Instituto Nacional de Salud Pública. Encuesta Nacional de Salud y Nutrición: resultados nacionales 2012. Síntesis ejecutiva. Cuernavaca (mx): Instituto Nacional de Salud Pública; 2012.

4. Instituto Nacional de Salud Pública. Encuesta Nacional de Salud y Nutrición: resultados nacionales 2012. Evidencia para la política pública en salud. Demanda y utilización de servicios de salud ambulatorios en el contexto de la cobertura universal. Cuernavaca (мx): Instituto Nacional de Salud Pública.

5. Knaul FM, Arreola-Ornelas H. Justicia financiera y gastos catastróficos en salud: impacto del Seguro Popular de Salud en México. Salud Publica Mex. 2005 Feb 2;47(supl 1): s54-65.

6. Zuluaga-Ramírez MM, Macedo-Ivanauskas T, Fagundes-Ferreira F, Rodrigues-Neto C. Impacto de la utilización de la red propia de una aseguradora privada sobre los costos de atención ambulatoria. Salud Publica Mex. 2015;57(5):426-32.https://doi.org/10.21149/ spm.v57i5.7622

7. Knaul F, Arreola-Ornelas H, Méndez-Carniado O. Protección financiera en salud: actualizaciones para México a 2014. Salud Publica Mex. 2016;58(3):341-50. https://doi. org/10.21149/spm.v58i3.7886

8. Pavón-León P, Reyes-Morales H, Martínez AJ, Méndez-Maín SM, Gogeascoechea-Trejo M del C, Blázquez-Morales MSL. Gasto de bolsillo en adultos mayores afiliados a un seguro público de salud en México. Gac Sanit. 2017 Jul;31(4):286-91. https://doi.org/10.1016/j. gaceta.2016.12.015

9. Sánchez Torres DA. Accesibilidad a los servicios de salud: debate teórico sobre determinantes e implicaciones en la política pública de salud. Rev Med Inst Mex Seguro Soc. 2017;55(1):82-9.

10. Knaul FM, Arreola-Ornelas H, Wong R, Lugo-Palacios DG, Méndez-Carniado O. Efecto del Seguro Popular de Salud sobre los gastos catastróficos y empobrecedores en México, 2004-2012. Salud Publica Mex. 2018 mar 23;60(2):130. https://doi.org/10.21149/9064

11. Gerdtham UG. Equity in health care utilization: further tests based on hurdle models and Swedish micro data. Health Econ. 1997;6(3):303-19. https://doi.org/10.1002/(sici)10991050(199705)6:3<303::aid-hec270>3.0.c0;2-p

12. McFadden D. Frontiers in econometrics. New York: Academic Press; 1973. p. 105-42.

13. Evans RG. Supplier-induced demand: some empirical evidence and implications. En: The economics of health and medical care. London: Palgrave Macmillan; 1974. p. 162-73.

14. Maddala GS. Limited-dependent and qualitative variables in econometrics. Cambridge, UK: Cambridge University Press; 1983.

15. Ben-Akiva ME and Lerman SR. Discrete choice analysis : theory and application to travel demand. London: The MIT Press; 1985. 
16. Becker GS, Murphy KM. A Theory of rational addiction. J Polit Econ. 1988 Aug;96(4):675700. https://doi.org/10.1086/261558

17. Wagstaff A. The demand for health: an empirical reformulation of the Grossman model. Health Econ. 1993;2(2):189-98. https://doi.org/10.1002/hec.4730020211

18. Clavero-Barranquero A, González-Álvarez L. Una revisión de modelos econométricos aplicados al análisis de demanda y utilización de servicios sanitarios. Rev Econ Pública. 2005; 73-(2/2005):129-62.

19. Finkelstein A, Stiglitz J, Arrow K. Moral hazard in health insurance. New York: Columbia University Press; 2014. https://doi.org/10.7312/columbia/9780231163804.001.0001

20. Grossman M. The demand for health: a theoretical and empirical investigation. New York: Columbia University Press; 1972.

21. Santos-Padrón H, Martínez-Calvo S, Martínez-López MC, Álvarez-Malpica IL. La demanda potencial y la oferta de servicios de salud para las enfermedades catastróficas en México. Rev Gerenc y Polit Salud. 2011;10(21):33-47.

22. Bautista-Arredondo S, Serván-Mori E, Colchero MA, Ramírez-Rodríguez B, Sosa-Rubí SG. Análisis del uso de servicios ambulatorios curativos en el contexto de la reforma para la protección universal en salud en México. Salud Publica Mex. 2014;56(1):18-31. https://doi.org/10.21149/spm.v56i1.7319

23. Gómez de León J, Bloom E, Bitran R, Dow W, Straffon B, Orozco M. La demanda de servicios de salud en México: un análisis econométrico. México: Conapo; 1995.

24. Zamudio A. La demanda por servicios médicos en México [documento de trabajo del CIDE 94]. México: CIDE; 1997.

25. Arreola $\mathrm{H}$, Soto H. Los determinantes de la no demanda de servicios para la salud. En: Knaul FM, Nigenda G, editores. Caleidoscopio de la salud: de la investigación a las políticas y de las políticas a la acción. México: Fundación Mexicana para la Salud; 2003. p. 173-83.

26. Valencia-Mendoza A, Bertozzi SM. A predictive model for the utilization of curative ambulatory health services in Mexico. Salud Publica Mex. 2008;50(5):397-407.

27. González-Block MA, Sauceda-Valenzuela AL, Santa Ana-Téllez Y. Factores asociados a la demanda de servicios para la atención del parto en México. Salud Publica Mex. 2010;52(5):416-23.

28. Hurley JE. The demand for health care. En: Health economics. London: McGraw-HillRyerson; 2010. p. 206-31.

29. Secretaría de Salud, Instituto Nacional de Salud Pública, Instituto Nacional de Estadística y Geografía. Encuesta Nacional de Salud y Nutrición (Ensanut) 2018. Ensanut; 2019.

30. Jiménez-Caballero E, Flores L, Peralta N. Políticas y programas que reducen los gastos catastróficos de salud en países de bajos y medianos ingresos. Rev Salud Pública Parag. 2018;8(2):57-65.

31. Oyarte M, Espinoza M, Balmaceda C, Villegas R, Cabieses B, Díaz J. Gasto de bolsillo en salud y medicamentos en Chile: análisis comparativo de los periodos 1997, 2007, y 2012. Value Heal Reg Issues. 2018 Dec;17:202-9. https://doi.org/0.1016/j.vhri.2018.09.006 
32. Petrera-Pavone M, Jiménez-Sánchez E. Determinantes del gasto de bolsillo en salud de la población pobre atendida en servicios de salud públicos en Perú, 2010-2014. Rev Panam Salud Publica. 2018;42:1-7. https://doi.org/10.26633/RPSP.2018.20

33. Giménez-Caballero E, Rodríguez JC, Ocampos G, Flores L. Composición del gasto de bolsillo en el sistema de salud del Paraguay. Mem Inst Investig Cienc Salud. 2017;15(3):64-72.

34. Aguilar-Palacio I, Carrera-Lasfuentes P, Solsona S, Sartolo MT, Rabanaque MJ. Utilización de servicios sanitarios en ancianos (España 2006-2012): influencia del nivel de salud y de la clase social. Aten Prim. 2016;48(4):235-43. https://doi.org/10.1016/j.aprim.2015.01.016

35. Arrivillaga M, Aristizábal JC, Pérez M, Estrada VE. Encuesta de acceso a servicios de salud para hogares colombianos. Gac Sanit. 2016;30(6):415-20. https://doi.org/10.1016/j. gaceta.2016.05.008

36. Beceyro AC, Aguilar-Álvarez CaP. Aspectos territoriales de la oferta-demanda de servicios de salud en el departamento Maipú (Mendoza), 2012-2015. Bol Estud Geogr. 2016;106:63-87.

37. Bernad-Vallés M, Maderuelo-Fernández JÁ, Moreno-González P. Necesidades de información sobre salud y enfermedad de usuarios de servicios sanitarios de atención primaria de Salamanca. At Prim. 2016;48(1):15-24. https://doi.org/10.1016/j.aprim.2015.01.011

38. Armijos-Bravo G, Camino-Mogro S. Inequidades socioeconómicas en el uso de servicios sanitarios de atención primaria en Ecuador. Estud Gerenc. 2017;33(144):292-301. https:// doi.org/10.1016/j.estger.2017.08.002

39. Dmytraczenko T, Almeida G, editores. Hacia la cobertura universal en salud y la equidad en América Latina y el Caribe: evidencia de países seleccionados. The World Bank; 2017. https://doi.org/10.1596/978-1-4648-1177-7

40. Gupta I, Dasgupta P. Demand for curative health care in rural India: choosing between private, public, and no care. New Delhi, India: National Council of Applied Economic Research; 2002.

41. Trías J. Determinantes de la utilización de los servicios de salud: el caso de los niños en la Argentina [internet]. La Plata: Universidad Nacional de La Plata; 2004 [citado 2019 ene 11]. Disponible en: http://www.depeco.econo.unlp.edu.ar/wp/wp-content/ uploads/2017/05/doc51.pdf

42. Knaul FM, Arreola-Ornelas H, Méndez-Carniado Ó, Brysin-Cahn C, Barofsky J, Maguire R, et al. Las evidencias benefician al sistema de salud: reforma para remediar el gasto catastrófico y empobrecedor en salud en México. Salud Publica Mex. 2007;49 (supl 1):s70-87.

43. Lara-Figueroa H. La calidad como determinante en los servicios de atención primaria en México [tesis de doctorado]. Flacso México; 2011.

44. Chemor Ruiz A, Ratsch A, Alamilla Martínez G. Mexico’s Seguro Popular: achievements and challenges. Health Systems Reform. 2018;4(3):194-202. https://doi.org/10.1080/232 88604.2018.1488505

45. Nigenda G, González-Robledo L, Juárez-Ramírez C, Adam T. Understanding the dynamics of the Seguro Popular de Salud policy implementation in Mexico from a complex adaptive systems perspective. Implement Sci. 2015;11(1). https://doi.org/10.1186/s13012016-0439-x 
46. Wagstaff A, Flores G, Smitz M, Hsu J, Chepynoga K, Eozenou P. Progress on impoverishing health spending in 122 countries: a retrospective observational study. Lancet Glob Health. 2018;6(2):e180-e192. https://doi.org/10.1016/S2214-109X(17)30486-2

47. Arenas E, Parker S, Rubalcava L, Teruel G. Evaluación del Programa del Seguro Popular del 2002 al 2005: impacto en la utilización de servicios médicos, en el gasto en salud y en el mercado laboral. Trimest Econ. 2015;82(328):807-45.

48. Luna M. Cuando el aseguramiento en salud no basta: ¿quiénes gastan más en servicios y atención a la saud? Coyunt Demogr. 2015;7:15-21.

49. Prada SI, Sarría MA, Guerrero R. Medición del gasto de bolsillo en salud usando la Encuesta Nacional de Calidad de Vida de Colombia (Measuring Out-of-Pocket Health Expenditure Using Colombia's Living Standards Measurement Studies). ssRn. 2016. https:// doi.org/10.2139/ssrn.2931192 